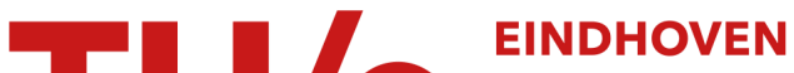 \\ UNIVERSITY OF \\ TECHNOLOGY
}

\section{Compositionality in the temporal logic of concurrent systems}

Citation for published version (APA):

Boer, de, F.S. (1990). Compositionality in the temporal logic of concurrent systems. (Computing science notes; Vol. 9015). Technische Universiteit Eindhoven.

Document status and date:

Published: 01/01/1990

\section{Document Version:}

Publisher's PDF, also known as Version of Record (includes final page, issue and volume numbers)

\section{Please check the document version of this publication:}

- A submitted manuscript is the version of the article upon submission and before peer-review. There can be important differences between the submitted version and the official published version of record. People interested in the research are advised to contact the author for the final version of the publication, or visit the $\mathrm{DOI}$ to the publisher's website.

- The final author version and the galley proof are versions of the publication after peer review.

- The final published version features the final layout of the paper including the volume, issue and page numbers.

Link to publication

\section{General rights}

Copyright and moral rights for the publications made accessible in the public portal are retained by the authors and/or other copyright owners and it is a condition of accessing publications that users recognise and abide by the legal requirements associated with these rights.

- Users may download and print one copy of any publication from the public portal for the purpose of private study or research.

- You may not further distribute the material or use it for any profit-making activity or commercial gain

- You may freely distribute the URL identifying the publication in the public portal.

If the publication is distributed under the terms of Article $25 \mathrm{fa}$ of the Dutch Copyright Act, indicated by the "Taverne" license above, please follow below link for the End User Agreement:

www.tue.nl/taverne

Take down policy

If you believe that this document breaches copyright please contact us at:

openaccess@tue.nl

providing details and we will investigate your claim. 
Compositionality in the temporal

logic of concurrent systems

by

F.S. de Boer

$90 / 15$

October, 1990 
COMPUTING SCIENCE NOTES

This is a series of notes of the Computing Science Section of the Department of Mathematics and Computing Science Eindhoven University of Technology.

Since many of these notes are preliminary versions or may be published elsewhere, they have a limited distribution only and are not for review.

Copies of these notes are available from the author or the editor.

Eindhoven University of Technology

Department of Mathematics and Computing Science

P.O. Box 513

$5600 \mathrm{MB}$ EINDHOVEN

The Netherlands

All rights reserved

Editors: prof.dr.M.Rem

prof.dr.K.M. van Hee 
Compositionality in the Temporal Logic of Concurrent Systems.

(extended abstract)

\author{
F.S. de Boer \\ Centre for Mathematics and Computer Science \\ P.O. Box 4079, 1009 AB Amsterdam, The Netherlands
}

\begin{abstract}
In this paper we develop a new method for describing in temporal logic in a compositional manner sequential composition, its iterated version (loops) and its interaction with (nested) parallelism. The logic we will use will be a linear time logic with as sole temporal operator the until. We will illustrate this method in the construction of a compositional proof system for a CSP-like language. We will prove this system to be sound and (relative) complete.
\end{abstract}

1980 Mathematics Subject Classification: 70405.

1985 CR Categories: F.3.1.

Key Words \& Phrases: Temporal Logic, Compositionality, Concurrency.

\title{
INTRODUCTION
}

Temporal logic formalizes reasoning about structures changing in time ([P1). We can apply temporal logic to program verification by interpreting time as a sequence of states, a state being a representation of the abstract machine by which our programs are run ([Pn]). One of the basic temporal operators is the (binary) until operator, denoted by $U$. The meaning of a temporal assertion $\psi_{1} U \psi_{2}$ can be described roughly as follows: $\psi_{2}$ is guaranteed to hold in the future and $\psi_{1}$ holds continuously until then. Given a temporal assertion $\psi$, program $S$, we define $\psi$ to be a specification of $S$, written as $\{S\} \psi$, if $\psi$ holds in every execution sequence of $S$. In this paper we address the problem of axiomatizing this notion.

In the literature there are given various axiomatizations in temporal logic of various (concurrent) programming languages ([BKP1], [L1], [MP], [NGO], [OG]). But a desirable property of an axiomatization is compositionality, by which we mean that the specification of a composite program is derivable from some specifications of its constituents. How to prove a specification of a program $S_{1} ; S_{2}$, the execution of which consists of an execution of $S_{1}$ followed by $S_{2}$, in a compositional manner, using specifications of $S_{1}$ and $S_{2}$, is however not yet answered in a satisfactory way.

There are developed until now essentially two ways to describe sequential composition in temporal logic: one of which consists of introducing location predicates, the other of introducing new temporal operators.

Introducing location predicates consists of adding to the specification language for each program $S$ the predicates $I N(S), A T(S), A F T E R(S)$ ([L2]). To evaluate these predicates we have to add a component to each state, a program counter, such that, for example, $I N(S)$ holds in a state if the program counter points to a location in $S$. Given these predicates we can formulate the following rule for sequential composition.

This research was partially sponsored by Esprit project 415: Parallel Architectures and Languages for AIP. 


$$
\frac{\left\{S_{1}\right\} \psi_{1},\left\{S_{2}\right\} \psi_{2}}{\left\{S_{1} ; S_{2}\right\} \psi_{1} \wedge\left(I N\left(S_{1}\right) U^{-} \psi_{2}\right)}
$$

The assertion $I N\left(S_{1}\right) U^{-} \psi_{2}$ will be true iff $I N\left(S_{1}\right) U \psi_{2}$ holds or $I N\left(S_{1}\right)$ holds throughout the future (this $U^{-}$operator can be easily expressed by the until operator). The assertion $\psi_{1} \wedge\left(I N\left(S_{1}\right) U^{-} \psi_{2}\right)$ describes a sequence in which $\psi_{1}$ holds and which can be decomposed in a (possibly infinite) prefix such that in every state of this prefix $I N\left(S_{1}\right)$ holds, that is: control is at some location of $S_{1}$, and $\psi_{2}$ holds in the suffix (possibly empty) determined by this prefix.

Note that for this rule to be sound we have to interpret $\{S\} \psi$ as follows: for every computation of $S$ $\psi$ holds in all those sequences which are obtained by appending an arbitrary sequence to this computation. If we interpret $\{S\} \psi$ as: $\psi$ holds in every computation of $S$, the above rule would be unsound. For example, if the statement $S_{1}$ does not change the value of some variable $x$ a valid specification of $S_{1}$, with respect to this interpretation, would be "the value of $x$ does not change", which can be expressed in temporal logic. But if the statement $S_{2}$ does change the value of $x$ then, obviously, this specification does not hold for $S_{1} ; S_{2}$.

The introduction of location predicates, however, will in general complicate the proof procedure. One needs in addition an axiomatization of these predicates. Furthermore, ideally a specification of a statement should specify only what it does, and not how it accomplishes its task. In a compositional proof system reasoning about the control structure should be carried out only in the proof that a statement meets a particular specification. That is, the control structure should be reflected by the structure of the axioms and rules of the proof system. Location predicates however introduce in the specification language the expressibility of the "fflow of control". In [BKP2], for example, to construct a fully abstract temporal semantics for a particular concurrent programming language these location predicates are dispensed with by restricting sequential composition to a head-tail construction: given an atomic action $a$ and a statement $S$ one can construct the statement $a ; S$. Applying our method for describing sequential composition would generalizes this fully abstract semantics to the language containing the usual notion of sequential composition.

Another way to describe sequential composition is introduced in [BKP1]: a new temporal operator, called the chop-operator, denoted by $C$, is defined such that the temporal assertion $\psi_{1} C \psi_{2}$ holds in a particular sequence if this sequence can be decomposed into a prefix in which $\psi_{1}$ holds and a suffix determined by this particular prefix in which $\psi_{2}$ holds. Now if $\psi_{1}$ describes $S_{1}$ and $\psi_{2}$ describes $S_{2}$ then $\psi_{1} C \psi_{2}$ will specify $S_{1} ; S_{2}$. Unfortunately this $C$ operator can greatly increase the complexity of the decision procedure (of the language at the propositional level), and will in general complicate the proof procedure ([HMM]).

We will show how one can describe sequential composition in a temporal logic with as sole temporal operator the until operator (by which all the other familiar temporal operators as "always", "sometimes", are expressible), the propositional level of this logic being decidable in exponential time ([HKP]), in a compositional manner in the context of a CSP-like language ([H]), without making use of location predicates.

Our method can be described roughly as follows. We specify a statement $S$ with respect to some possible continuation of $S$. In our assertion language (the language for the description of sequences of states) this continuation will be denoted by some particular predicate variable. Let $Y$ be this predicate variable. We define a specification $\{S\} \psi(Y)$ to be true iff for every (infinite) sequence consisting of a prefix which is a computation of $S \psi(Y)$ holds in this sequence when $Y$ is interpreted as the suffix determined by this computation. In this framework it can be shown that if $\left\{S_{1}\right\} \psi_{1}(Y)$ and $\left\{S_{2}\right\} \psi_{2}(Y)$ are valid specifications then $\psi_{1}\left[\psi_{2} / Y\right]$ will specify $S_{1} ; S_{2}$. Here $\psi_{1}\left[\psi_{2} / Y\right]$ denotes the result of substituting $\psi_{2}$ for the free occurrences of $Y$ in $\psi_{1}$. Furthermore we will show how one can describe iterated sequential composition (loops) and the behaviour resulting from the interaction between sequential and parallel composition in this framework.

This paper is organized as follows. In the first section we describe the programming language the proof theory of which we will study. We will define a semantics for this language in the second section. In the third section we introduce the assertion language used to describe sequences of states and 
describe its semantics. Then we present in section four the proof system. In the sections five and six we prove the soundness and completeness of the system. In the last section we discuss how to extend our method for a CSP-like language containing nested parallelism.

\section{The Programming Language}

Given the sets, $L$, a set of function and predicate symbols, $V A R=\{x, \ldots\}$, the set of program variables, $C H A N=\{c, \ldots\}$, the set of channel names, the class of statements, denoted by $S T A T$, is defined as follows:

$$
\begin{gathered}
S::=x:=t \\
\mid \text { skip } \\
|c ! t| c ? y \\
\mid S_{1} ; S_{2} \\
\mid \unrhd_{i=1}^{n} b_{i} \rightarrow S_{i} \\
\mid \star \square_{i=1}^{n} b_{i} \rightarrow S_{i}
\end{gathered}
$$

Where $t$ denotes a term built up from program variables and function symbols and $b$ denotes a boolean expression built up from terms, predicate symbols, and the usual logical connectives like $\wedge, \vee, \neg, \ldots$

The execution of an assignment statement $x:=t$ consists of assigning the value of the term $t$ to the variable $x$. The execution of a skip-statement does not change the state. The statements $c ! t, c ? y$ are called IO commands. The execution of an output statement $c ! t$ is synchronized with an execution of an input statement $c ? y$, the result of this synchronization being that of the assignment $y:=t$. The statement $S_{1} ; S_{2}$ denotes as usual the sequential composition of $S_{1}$ and $S_{2}$. The last two statements are called the alternative command and the iterative command. These commands are generalizations of the if - then - else - $f$ and the while - do-od construct ([D]).

The set of programs is defined as follows:

$$
P::=S_{1}\|\cdots\| S_{n}
$$

where $\operatorname{VAR}\left(S_{i}\right) \cap \operatorname{VAR}\left(S_{j}\right)=\varnothing,(1 \leqslant i \neq j \leqslant n)$, and every channel occurring in $P$ connects only two processes, one of which is only allowed to read from it, the other only to write to it.

This restriction on channels is introduced in [ZRE] to obtain a compositional Hoare-style proof system for a CSP-like language containing nested parallelism.

$\operatorname{VAR}(S)$ denotes the set of program variables occurring in $S$. CHAN $(S)$ denotes the set of channels occurring in $S$.

Execution of a program $P \equiv S_{1}\|\cdots\| S_{n}$ consists of the parallel execution of the statements $S_{i}$.

\section{Semantics}

In this section we define a semantics for the programming language defined in the previous section. We fix some set $L$ of function and predicate symbols, and some interpretation $I=(D A T A, J)$ for $L$, where $D A T A$ is some domain of values and $J$ some interpretation of the function, predicate symbols of $L$ (the set $L$ and the interpretation $I$ we assume to be fixed throughout the paper). To define the semantics we need the following definitions:

DeFINITION 2.1:

We define $\Sigma$, the set of states, with typical element $\sigma$, as follows:

$$
\Sigma=(V A R \rightarrow D A T A) \times\left(C H A N \rightarrow D A T A^{*}\right)
$$


A state consists of two components. The first component gives values to the program variables. The second component associates with each channel the sequence of data transferred on it, DATA* denoting the set of finite sequences of elements of DATA.

The value of a variable $x$ with respect to a state $\sigma$ we denote by $\sigma(x)$, and the value of a channel $c$ with respect to a state $\sigma$ will be denoted by $\sigma(c)$. This will cause no confusion because we assume $C H A N \cap V A R$ to be empty.

Definition 2.2.:

We define the set of contexts $C O N$, with typical element $C$, as follows:

$$
C O N=\{(V, W): V \subseteq V A R, W \subseteq C H A N\}
$$

With each statement $S$ we associate a $\operatorname{CON}(S) \in C O N$ such that the first component contains all the program variables of $S$, the second all the channels occurring in $S$. More precisely, $\operatorname{CON}(S)=(\operatorname{VAR}(S), C H A N(S))$, where $\operatorname{VAR}(S)$ denotes the set of variables occurring in $S$ and $C H A N(S)$ denotes the set of channels of $S$.

\section{DEFINITION 2.3.:}

Given a context $C$ we define the set of local states $\Sigma(C)$, with typical element $s$, as follows:

$$
\Sigma(C)=(V \rightarrow D A T A) \times\left(W \rightarrow D A T A^{*}\right) \text {, where } C=(V, W) .
$$

$\Sigma(C)$ denotes the set of local states specified by the context $C$. Given $\sigma \in \Sigma$ we define $\sigma(C) \in \Sigma(C)$, the restriction of $\sigma$ to $\Sigma(C)$ as follows:

$$
\begin{aligned}
& \sigma(C)(x)=\sigma(x), x \in V \\
& \sigma(C)(c)=\sigma(c), c \in W
\end{aligned}
$$

The set of finite sequences of local states of $\Sigma(C)$ we denote by $\Sigma(C)^{*}$, the set of infinite sequences of local states of $\Sigma(C)$ we denote by $\Sigma(C)^{\omega}$, and the union of these sets, with typical element $p$, is denoted by $\Sigma(C)^{\infty}$.

\section{DEFINITION 2.4.:}

Let $\rho_{1}, \rho_{2} \in \Sigma(C)^{\infty}$. We define $\rho_{1} \otimes \rho_{2}$, the fusion of $\rho_{1}$ and $\rho_{2}$, as follows:

$$
\begin{aligned}
\rho_{1} \otimes \rho_{2} & =\rho_{1} \text { if } \rho_{1} \in \Sigma(C)^{\omega} \\
& =\left\langle s_{1}, \ldots, s_{n}, s_{n+1}, \ldots>\text { if } \rho_{1}=\left\langle s_{1}, \ldots, s_{n}\right\rangle \text { and } \rho_{2}=\left\langle s_{n}, \ldots\right\rangle\right.
\end{aligned}
$$

undefined, otherwise

\section{DEFINITION 2.5.:}

We next define the operation last: $\Sigma(C)^{\infty} \rightarrow \Sigma(C)$ :

$$
\text { last } \begin{aligned}
(\rho) & =s_{n} \text { if } \rho=\left\langle s_{1}, \ldots, s_{n}\right\rangle \\
& =s \text { if } \rho \in \Sigma(C)^{\omega},
\end{aligned}
$$

In this definition $s$ is some arbitrary fixed local state.

Definition 2.6.:

Let $X \subseteq \Sigma(C)^{\infty}, \rho \in \Sigma(C)$. We define:

$$
\begin{aligned}
& \rho \otimes X=\left\{\rho \otimes \rho_{1}: \rho_{1} \in X\right\} \text { if } X \neq \varnothing \text { or } \rho \in \Sigma(C)^{*} \\
& \rho \otimes X=\{\rho\} \text { otherwise. }
\end{aligned}
$$

$\rho \otimes X$ denotes the set of sequences resulting from fusing $\rho$ with an arbitrary sequence of $X$. 
DEFINITION 2.7.:

Given $s \in \Sigma(C)$, we define:

$$
\begin{aligned}
s^{0} & =\epsilon \text { (the empty sequence) } \\
s^{n+1} & =s^{n_{0}}<s>
\end{aligned}
$$

The set of (finite and infinite) sequences of global states, with typical element $\tau$, we denote by $\Sigma^{\infty}$.

DEFINITION 2.8.:

Let $\tau=\left\langle\sigma_{n}\right\rangle_{n} \in \Sigma^{\infty}\left(\Sigma^{\infty}=\Sigma^{*} \cup \Sigma^{\omega}\right)$. For $l \leqslant n \leqslant|\tau|$, where $|\tau|$ denotes the length of $\tau, \tau(n)$ denotes the $n^{\text {th }}$ state of $\tau$. For $1 \leqslant n<m \leqslant \omega$ we define $\tau[n, m]=<\tau(k+1)>_{n-1<k<m}: \tau[n, m]$ denotes the subsequence of $\tau$ determined by $n$ and $m$. Given a context $C$ we define $\tau(C)=\left\langle\sigma_{n}(C)\right\rangle_{n}: \tau(C)$ denotes the restriction of the sequence $\tau$ to $\Sigma(C)^{\infty}$.

\section{DEFINITION 2.9.:}

The value of a term $t$ in a state $s$, where $s \in \Sigma(C)$, with $C=(V, W)$ such that $V A R(t) \subseteq V$, we denote by [ $t](s)$. We define the truth of a boolean expression $b$, with $V A R(b) \subseteq V$, in a local state $s \in \Sigma(C)$, assuming $C=(V, W)$, in the usual way. Notation: $s \neq b$. $(V A R(t), V A R(b)$ denotes the set of program variables occurring in $t, b$, respectively.)

DEFINITION 2.10.:

Given a local state $s$, the local state obtained by assigning the value $d$ to a variable $x$ we denote by $s\{d / x\}$, and the local state resulting from assigning the sequence of data $d^{*}$ to a channel $c$ is denoted by $s\left\{d^{*} / c\right\}$. In general, given a function $f \in A \rightarrow B, f\{b / a\}$ is defined as follows:

$$
\begin{aligned}
f\{b / a\}(c) & =f(c), c \neq a \\
& =b, c=a
\end{aligned}
$$

Finally we are ready to define the semantics of the programming language defined above. We define $M(C): S T A T(C) \rightarrow \Sigma(C) \rightarrow P\left(\Sigma(C)^{\infty}\right), \quad$ where $\quad S T A T(C)=\{S: \operatorname{CON}(S) \subseteq C\} \quad$ (let $C_{1}=\left(V_{1}, W_{1}\right), C_{2}=\left(V_{2}, W_{2}\right)$ then $C_{1} \subseteq C_{2}$ iff $V_{1} \subseteq V_{2}$ and $\left.W_{1} \subseteq W_{2}\right)$.

$M(C)(S)(s)$ will deliver as value all the execution sequences of $S$ starting from state $s$. The context $C$ restricts the semantic description of $S$ to the variables and channels specified by it.

$$
\begin{aligned}
& M(C)(x:=t)(s)=\left\{s^{n_{0}}<s_{1}>: 1 \leqslant n<\omega \wedge s_{1}=s\{[t](s) / x\}\right\} \\
& M(C)(s k i p)(s)=\left\{<s^{n}>: 1<n<\omega\right\} \\
& M(C)(c ! t)(s)=\left\{s^{n_{\circ}}<s_{1}>: 1 \leqslant n<\omega \wedge s_{1}=s\{s(c) \circ<\llbracket t \rrbracket(s)>/ c\}\right\} \\
& M(C)(c ? y)(s)=\bigcup_{d \in D A T A}\left\{s^{n_{\circ}}<s_{1}>: 1 \leqslant n<\omega \wedge s_{1}=s\{s(c) \circ<d>/ c\}\{d / y\}\right\} \\
& M(C)\left(S_{1} ; S_{2}\right)(s)=\bigcup_{\rho \in M(C)\left(S_{1}\right)(s)} \rho \otimes M(C)\left(S_{2}\right)(\text { last }(\rho)) \\
& M(C)\left(\square_{i} b_{i} \rightarrow S_{1}\right)(s)=\bigcup_{i \in \mathrm{I}} M(C)\left(S_{i}\right)(s), \mathrm{I}=\left\{i: s \vDash b_{i}\right\} \\
& M(C)\left(\star \square_{i} b_{i} \rightarrow S_{i}\right)(s)=\nu F(s), \\
& \text { where } F: D \rightarrow D, D=\Sigma(C) \rightarrow P\left(\Sigma(C)^{\infty}\right) \text { is defined such that: } \\
& F(f)(s)=M(C)(s k i p)(s) \text { if } s \vDash \wedge_{i} \neg b_{i} \\
& =\bigcup_{i \in \mathrm{I}} \bigcup_{\rho \in M(C)\left(S_{)}\right)(s)} \rho \otimes f(\text { last }(\rho)) \text { if } \mathrm{I}=\left\{i: s \vDash b_{i}\right\} \neq \varnothing
\end{aligned}
$$


Here $\nu F$ denotes the greatest fixed point of $F$.

Some comments are in order here. The meaning of the iterative construct is well defined because of the following lemma. This lemma is a weak form of a theorem due to Tarski ([T]), which generalizes an earlier result of Knaster and Tarski.

LEMMA 2.11:

Let $L$ be a complete lattice, $T$ its upperbound, $f$ a monotonic operator on $L$.

There exists a greatest fixed point of $f$, which we denote by $\nu f$. This fixed point can be characterized in the following two ways.

$$
\begin{aligned}
& \nu f=l . u . b .\{x \in L: x \leqslant f(x)\} \text { and } \\
& \nu f=f^{\alpha}(T), \alpha \text { the least ordinal of cardinality greater than that of } L
\end{aligned}
$$

where

$$
\begin{aligned}
& f^{0}(T)=T, \\
& f^{\beta+1}(T)=f\left(f^{\beta}(T)\right), \\
& f^{\alpha}(T)=\underset{\beta<\alpha}{\cap} f^{\beta}(T), \alpha \text { a limit ordinal. }
\end{aligned}
$$

Proof : see ([T]).

Now the following ordering turns $D=\Sigma(C) \rightarrow P\left(\Sigma(C)^{\infty}\right)$ into a complete lattice. For $f, g \in D$ we define $f \leqslant g$ iff for every $s \in \Sigma(C)$ we have $f(s) \subseteq g(s)$, furthermore we define $f \cap g, f \cup g$ as follows: for every $s \in \Sigma(C)$ we have $(f \cap g)(s)=f(s) \cap g(s)$ and $(f \cup g)(s)=f(s) \cup g(s)$. It is easy to check that $F$ is monotonic on this complete lattice $D$.

We introduced in the meaning of the assignment statement the notion of finite stuttering because in our temporal logic, to be defined in the next section, we can not describe the assignment as taking place in one unit of time.

An output statement $c ! t$ is modelled as an assignment $c:=c^{\circ}<t>$, the function symbol $\circ$ being interpreted as concatenation. An input statement $c$ ? $y$ is modelled as: $y:=? ; c:=c^{\circ}<y>$. Execution of the statement $y:=$ ? consists of assigning some random value to $y$.

The finite stuttering present in the meaning of the IO statements models synchronization. Note that we force this synchronization to take place, thus abstracting from possible deadlock behaviour. We abstract from deadlock behaviour to focus our attention on the main problem: a compositional proof system. To incorporate deadlock behaviour we have to allow in the semantics of IO statements infinite stuttering. Communications then can be enforced to take place by requiring computation sequences to satisfy some fairness assumptions. To formulate these fairness assumptions we have to add to a state a component which for each channel records if some process is ready to write to or to read from it ([BKP1]).

Note that we also abstract from the failure due to the guards of some alternative construct being false.

In the definition of the parallel composition, see below, we merge only those local computations which are in agreement with respect to the choices made in the evaluation of the input statements.

We lift $M(C)$ to $O(C): S T A T \rightarrow P\left(\Sigma^{\infty}\right)$ as follows:

$$
\begin{aligned}
& O(C)(S)=\varnothing \text { if } \neg C O N(S) \subseteq C . \\
& O(C)(S)=\{\tau: \tau(C) \in M(C)(S)(\tau(1)(C))\} \text { otherwise. }
\end{aligned}
$$

So $O(C)(S)$ consists of all those sequences such that the restriction of it to the context $C$ is an element of $M(C)(S)(s)$, where $s$ is the restriction of the initial state to $C$. Finally we define: 


$$
O\left(S_{1}\|\cdots\| S_{n}\right)=\bigcap_{i} O\left(C_{i}\right)\left(S_{i} ; \text { halt }\right)
$$

where $C_{i}=\operatorname{CON}\left(S_{i}\right)$ and halt is an abbreviation of $\star \square$ true $\rightarrow$ skip. Note that the semantics of programs consists only of infinite sequences.

\section{The Assertion LANGuage}

In this section we will define the language used to describe sequences of states. We assume given the following types: $V A L, I N T, V A L^{\star}$. An element of our domain of values will be of type $V A L$. A sequence of data will be of type $V A L^{*}$. The type of integers we denote by $I N T$. We will use $a$ to range over this set of types. For each type $a$ the set of logical variables of type $a$ we denote by $L V A R^{a}$. For different types $a, a^{\prime}$ we have $L V A R^{a} \cap L V A R^{a^{\prime}}=\varnothing$. We next define the set terms $t_{a}$ of type $a$.

\section{DEFINITION 3.1.:}

We define:

$$
\begin{aligned}
& t_{a}::=z_{a}, z_{a} \in L V A R^{a} \\
& \mid x, x \in V A R, a=V A L \\
& \mid c, c \in \mathrm{CHAN}, a=V A L^{*} \\
& \ldots
\end{aligned}
$$

Given the set of predicate variables $P R E D V$, with typical element $X$, the set of assertions is defined as follows:

DEFINITION 3.2.:

We define:

$$
\begin{aligned}
\psi::=t_{a}=t^{\prime}{ }_{a}\left|t_{a} \leqslant t^{\prime}{ }_{a}, a=I N T\right| \cdots \\
\mid \exists z \psi, \psi \text { a state assertion, } z \text { a logical variable } \\
|X, X \in P R E D V| \neg \psi \mid \psi_{1} \wedge \psi_{2} \\
\mid L E T z_{a}=t_{a} I N \psi N I \\
\mid \psi_{1} U \psi_{2} \\
\mid \nu X \psi, X \text { occurring positively in } \psi \text { (in the scope of an even number of negations) }
\end{aligned}
$$

Note that we allow the usual quantification only over socalled state assertions, i.e., assertions containing no temporal operators. Quantification over an arbitrary assertion is only allowed by means of the $L E T$ - construct. The assertion $\nu X \psi$ will be interpreted as the greatest fixed point of $\psi, \psi$ viewed as a predicate transformer in $X$. We introduce the following abbreviations: $\psi_{1} \vee \psi_{2} \equiv \neg\left(\neg \psi_{1} \wedge \neg \psi_{2}\right)$, $\psi_{1} \rightarrow \psi_{2} \equiv \neg \psi_{1} \vee \psi_{2}, \quad \quad \square \psi \equiv \neg($ true $U \neg \psi), \quad \psi_{1} U^{-} \psi_{2} \equiv\left(\psi_{1} U \psi_{2}\right) \vee \square \psi$, LET $z_{1}, \ldots, z_{n}=t_{1}, \ldots, t_{n} I N \psi N I \equiv L E T z_{1}=t_{1} I N \cdots L E T z_{n}=t_{n} I N \psi N I \cdots N I$. The sequence $\neg, \wedge, \vee, \rightarrow, \square, U$ exhibits the connectives of the logic in decreasing order of binding priority.

We will evaluate an assertion $\psi$ in a logical environment (a function assigning values to logical variables), a predicate environment (a function which interprets predicate variables) and with respect to a pair $\langle\tau, n\rangle, \tau \in \Sigma^{\omega}, n$ a natural number. The idea is that the number $n$ in $\langle\tau, n\rangle$ denotes the $n^{\text {th }}$ state of $\tau$, which will be considered as the present. Basic assertions, for example, will be evaluated with respect to $\tau(n)$, the $n^{\text {th }}$ state of $\tau$. 
In the sequel the notation $\left\langle\tau, n>\right.$ will imply that $\tau \in \Sigma^{\omega}$, i.e., that $\tau$ is an infinite sequence.

DEFINITION 3.3.:

The set of logical environments, with typical element $\gamma$, is defined as follows:

$\prod_{a} L V A R^{a} \rightarrow D^{a}$

Here $\Pi$ denotes the generalized cartesian product. furthermore we define $D^{I N T}=\mathbb{N}, D^{V A L}=D A T A, D^{I N T^{*}}=\mathbb{N}^{*}$ and $D^{V A L^{*}}=D A T A^{*}$.

Definition 3.4.:

The set of predicate environments, with typical element $\eta$, is defined as follows:

$$
P R E D V \rightarrow P\left(\Sigma^{\omega} \times \mathbb{N}\right)
$$

Elements of $P\left(\Sigma^{\omega} \times \mathbf{N}\right)$ will be denoted by $\boldsymbol{\theta}, \boldsymbol{\theta}^{\prime}, \ldots$ Singleton sets of $P\left(\Sigma^{\omega} \times \mathbf{N}\right)$ including the empty set will be denoted by $\theta, \theta^{\prime}, \ldots$.

Next we define $\langle\tau, n\rangle, \gamma, \eta \vDash \psi$, the truth of the assertion $\psi$ with respect to the environments $\gamma, \eta$ and the sequence $\langle\tau, n\rangle$, by induction on the complexity of $\psi$.

\section{DEFINITION 3.5.:}

We treat the following cases:

$\bullet<\tau, n>, \gamma, \eta \vDash X$ iff $<\tau, n>\in \eta(X)$

- $\left\langle\tau, n>, \gamma, \eta \vDash L E T z_{a}=t_{a} I N \psi N I\right.$ iff $\left\langle\tau, n>, \gamma\left\{\left\lfloor t_{a} \rrbracket(\gamma)(\tau(n)) / z_{a}\right\}, \eta \vDash \psi\right.\right.$

where $\left[t_{a} \rrbracket(\gamma)(\tau(n))\right.$ denotes the result of evaluating $t_{a}$ in the logical environment $\gamma$ with respect to the $n^{\text {th }}$ state of the sequence $\tau$.

- $\left\langle\tau, n>, \gamma, \eta \vDash\left(\psi_{1} U \psi_{2}\right)\right.$ iff there exists a $m>n$ such that

$<\tau, m>, \gamma, \eta \vDash \psi_{2}$ and for all $n \leqslant k<m$ we have $<\tau, k>, \gamma, \eta \vDash \psi_{1}$

- $\langle\tau, n>, \gamma, \eta \vDash(\nu X \psi)$ iff $<\tau, n>, \gamma, \eta\{\Theta / X\} \vDash \psi$

where $\boldsymbol{\Theta}=\cup\left\{\boldsymbol{\Theta}^{\prime}: \boldsymbol{\Theta}^{\prime} \subseteq \mathbb{I} \psi \mathbf{]}(\gamma)\left(\eta\left\{\boldsymbol{\Theta}^{\prime} / X\right\}\right\}\right)$, with $[\psi](\gamma)(\eta)=\{\langle\tau, n>:\langle\tau, n>, \gamma, \eta \vDash \psi\}$.

With respect to the last clause we require that $X$ occurs positively in $\psi$, that is, only in the scope of an even number of negations. In that case $\psi$ can be viewed as a monotonic operator on the complete lattice $P\left(\Sigma^{\omega} \times \mathbb{N}\right)$ (ordered with respect to set inclusion), and the set $\Theta$ as its greatest fixed point (see lemma 2.1.: substitute $P\left(\Sigma^{\omega} \times \mathbf{N}\right)$ for $\boldsymbol{L}$ and interpret $f$ as $\left.\left.f(\boldsymbol{\theta})=\llbracket \psi\right](\gamma)(\eta\{\Theta / X\})\right)$.

Next we define the notion of validity of an assertion $\psi$, written as $\vDash \psi$. To this end we fix some predicate variable $Y$. Now we define $\psi$ to be valid iff for every (infinite) sequence and suffix of this sequence $\psi$ holds in this sequence when the predicate variable $Y$ is interpreted as this suffix. More precisely: We define an assertion $\psi$ to be valid if for an arbitrary logical environment $\gamma$, predicate environment $\eta$, and sequence $\langle\tau, n\rangle$ such that $\eta(Y)=\{<\tau, m>\}$, for some $n \leqslant m \leqslant \omega$, where we define $\{\langle\tau, \omega\rangle\}=\varnothing,\langle\tau, n\rangle, \gamma, \eta \vDash \psi$ holds. To formalize this definition we need the following:

DEFINITION 3.6.:

Let $X \subseteq \Sigma^{\infty}, \tau \in \Sigma^{\omega}$. We define:

$$
\operatorname{CONT}(X,<\tau, n>)=\{\{<\tau, m>\}: n \leqslant m \leqslant \omega \wedge \tau[n, m] \in X\}
$$

where we define $\{\langle\tau, \omega\rangle\}=\varnothing$. 
$\operatorname{CONT}(X,<\tau, n>)$ essentially denotes all the suffixes of the sequence $\tau[n, \omega]$ such that the prefix of $\tau[n, \omega]$ determined by such a suffix belongs to this set of sequences $X$.

In the next definition we define formally the notion of validity as described above.

DEFINITION 3.7.:

We define

$$
\vDash \psi \text { iff } \forall \gamma, \eta,\left\langle\tau, n>, \theta \in \operatorname{CONT}\left(\Sigma^{\infty},<\tau, n>\right):<\tau, n>, \gamma, \eta\{\theta / Y\} \vDash \psi\right.
$$

Note that we have $\vDash \square \neg Y \vee(\neg Y U Y \wedge \square \neg Y)$. This axiom expresses that the predicate variable $Y$ denotes some unique suffix. In fact the following is the case. Define: $\mid=\psi$ iff for every $\langle\tau, n\rangle, \gamma, \eta$ we have $\langle\tau, n>, \gamma, \eta \vDash \psi$, so $| \vDash$ denotes the usual notion of validity. Suppose + completely axiomatizes $\mid k$.

Then $\vdash$ together with the axiom $\square \neg Y \vee(\neg Y U Y \wedge \square \neg Y)$ completely axiomatizes $\approx$ because of the following lemma.

LEMMA 3.8.:

For an arbitrary assertion $\psi$ we have

$$
\vDash \psi \text { iff } \mid \vDash \square \neg Y \vee(\neg Y U(Y \wedge \square \neg Y)) \rightarrow \psi
$$

Proof :

$\Rightarrow$ : Let $\gamma, \eta,<\tau, n>$ such that

$$
<\tau, n>, \gamma, \eta \vDash \square \neg Y \vee(\neg Y U Y \wedge \square \neg Y)(*)
$$

We have

$$
<\tau, n>, \gamma, \eta \vDash \psi \text { iff }<\tau, n>, \gamma, \eta^{\prime} \vDash \psi
$$

where $\eta^{\prime}(Y)=\eta(Y) \cap\{<\tau, m>: n \leqslant m<\omega\}$, and with respect to all other predicate variables $\eta^{\prime}$ agrees with $\eta$ (a proof of this is straightforward but tedious). But $\left(^{*}\right)$ then implies that for some $\theta \in \operatorname{CONT}(<\tau, n>)$ we have $\eta^{\prime}(Y)=\theta$. Now $<\tau, n>, \gamma, \eta\{\theta / Y\} \vDash \psi$ follows directly from $\vDash \psi$.

$\Leftarrow$ : Evident

In the sequel we abbreviate $\operatorname{CONT}\left(\Sigma^{\infty},<\tau, n>\right)$ to $\operatorname{CONT}(<\tau, n>)$.

A specification will be a formula $\{S\}_{C} \psi$ such that $\operatorname{CON}(S) \subseteq C=(V, W)$ (pointwise containment), $V A R(\psi) \subseteq V, C H A N(\psi) \subseteq W$, and $Y$ occurs positively in $\psi .(V A R(\psi)$ and $C N A N(\psi)$ denote the set of program variables and channels which occur in $\psi$.) The truth of such a specification is defined as follows:

DEFINITION 3.9.:

We define:

$$
\vDash\{S\}_{C} \psi \text { iff } \forall \gamma, \eta,<\tau, n>, \theta \in \operatorname{CONT}(O(C)(S),<\tau, n>):<\tau, n>, \gamma, \eta\{\theta / Y\} \vDash \psi
$$

Intuitively: A specification $\{S\}_{C} \psi$ holds iff $\psi$ holds in every sequence consisting of a prefix which is a computation of $S$ when $Y$ is interpreted as the suffix of this sequence determined by this particular prefix, that is, when $Y$ is interpreted as the continuation of this computation of $S$.

Programs are specified by formulas of the form $\{P\} \psi$, the semantics of which is given by the following definition:

Definition 3.10.: 
We define:

$$
\vDash\{P\} \psi \text { iff } \forall \gamma, \eta,<\tau, n>\text { such that } \tau[n, \omega] \in O(P) \text { we have }\langle\tau, n>, \gamma, \eta \vDash \psi
$$

We assume that $\psi$ does not contain free occurrences of the predicate variable $Y$. This assumption makes sense because at the top level we need not reason about possible continuations.

\section{The Proof System}

In this section we present the proof system for specifications as defined in the previous section. The axioms and rules of this system will closely mirror the semantic definitions of section 2 .

\section{INVARIANCE}

$$
\{S\}_{C} L E T z=t I N z=t U z=t \wedge Y N I
$$

Here $S$ is an atomic statement and if $C=(V, W)$ then $t \in(V \cup W) \backslash$ change $(S)$,

with change $(c ! t)=\{c\}$,

$$
\begin{aligned}
& \text { change }(c ! t)=\{c\}, \\
& \text { change }(c ? y)=\{c, y\}, \\
& \text { change }(x:=t)=\{x\}, \\
& \text { change }(\text { skip })=\varnothing .
\end{aligned}
$$

This axiom states the invariance of the value of the term $t$ during the computation of the atomic statement $S$. Note how this $L E T$ - construct is used to "freeze" the initial value of $t$. The continuation of this computation is denoted by the variable $Y$.

\section{ASSIGNMENT}

$$
\{x:=t\}_{C} \text { LET } z=x I N z=x \quad U x=t[z / x] \wedge Y N I
$$

This axiom describes the result of the assignment $x:=t$ on the variable $x$. The initial value of $x$ is stored in the logical variable $z$. After the execution of $x:=t$ the value of $x$ can then be described by the term $t[z / x]$. The continuation of the execution of the assignment statement is denoted by the predicate variable $Y$.

\section{OUTPUT}

$$
\{c ! t\}_{C} L E T z=c I N z=c U c=z^{\circ}<t>\wedge Y N I
$$

This axiom describes the effect of the output statement $c ! t$ as adding the value of the term $t$ to the sequence of data transferred on the channel $c$.

INPUT

$$
\{c ? y\}_{C} L E T z_{1}, z_{2}=c, y \quad I N z_{1}=c \wedge z_{2}=y \quad U c=z_{1}^{\circ}<y>\wedge Y N I
$$

Note that this axiom states nothing about the value of the variable $y$ after the execution of $c$ ? $y$. It states only that after the execution of $c ? y$ a certain relation must hold between $c$ and $y$. This axiom describes the effect of the input statement $c$ ? $y$ as assigning some arbitrary value to the variable $y$ and adding this value to the sequence of data transferred on the channel $c$. The logical variables $z_{1}$ and $z_{2}$ are used to freeze the initial values of the channel $c$ and the variable $y$. 


\section{SEQUENTIAL Composition}

$$
\frac{\left\{S_{1}\right\}_{C} \psi_{1} ;\left\{S_{2}\right\}_{C} \psi_{2}}{\left\{S_{1} ; S_{2}\right\}_{C} \psi_{1}\left[\psi_{2} / Y\right]}
$$

In this rule $\psi_{1}\left[\psi_{2} / Y\right]$ denotes the substitution of $\psi_{2}$ for $Y$ in $\psi_{1}$.

This substitution can be performed only if no free occurrences of predicate and logical variables of $\psi_{2}$ get bound. If this is the case we first have to rename some bound predicate and logical variables of $\psi_{1}$. The intuition behind this rule can be described as follows. If $k\left\{S_{i}\right\}_{C} \psi_{i}$ then one can view $\psi_{i}$ as describing some computation of $S_{i}$ followed by some continuation denoted by the predicate variable $Y$. But in the context of $S_{1} ; S_{2}$ occurrences of $Y$ in $\psi_{1}$, denoting some continuation of $S_{1}$, will denote a sequence consisting of a computation of $S_{2}$, i.e., a sequence satisfying $\psi_{2}$ when $Y$ in $\psi_{2}$ is interpreted as the continuation of $S_{1} ; S_{2}$. Now we can make occurrences of $Y$ in $\psi_{1}$ refer to sequences satisfying $\psi_{2}$ by substituting $\psi_{2}$ for $Y$ in $\psi_{1}$.

\section{Cholce}

We have the following rule for the alternative command:

$$
\frac{\left\{S_{i}\right\}_{C} \psi_{i}}{\left\{\square_{i} b_{i} \rightarrow S_{i}\right\}_{C} \underset{i}{\vee}\left(b_{i} \wedge \psi_{i}\right)}
$$

\section{ITERATION}

The iterative command is described by the following rule:

$$
\frac{\left\{S_{i}\right\}_{C} \psi_{i},\{\text { skip }\}_{C} \psi}{\left\{\star \square_{i} b_{i} \rightarrow S_{i}\right\} \nu X\left(\bigvee_{i}\left(b_{i} \wedge \psi_{i}[X / Y]\right) \vee\left(\wedge_{i} \neg b_{i} \wedge \psi\right)\right)}
$$

Here $X$ is a fresh predicate variable.

The idea of this rule can be described as follows. The predicate variable $Y$ in $\psi_{i}$ denotes some continuation of $S_{i}$. But in the context of ${ }_{i} b_{i} \rightarrow S_{i}$ the continuation of $S_{i}$ consists of an execution of ${ }^{*} \square_{i} b_{i} \rightarrow S_{i}$. This can be described by applying the fixed point operator to a fresh predicate variable which is substituted for $Y$ in $\psi_{i}$. Note that the assertion $\psi$, which specifies the statement skip, is used to describe finite stuttering.

\section{Parallel Composition}

We have the following rule for programs:

$$
\frac{\left\{S_{i} ; \text { halt }\right\}_{C_{i}} \psi_{i}}{\left\{S_{1}\|\cdots\| S_{n}\right\} \wedge_{i} \psi_{i}[\text { false } / Y]}
$$

where $C_{i}=\operatorname{CON}\left(S_{i}\right)$.

We may substitute false for $Y$ in $\psi_{i}$ because at this level there are no continuations. Note that freedom from interference is quaranteed by the syntactic restriction that $\operatorname{VAR}\left(\psi_{i}\right) \subseteq \operatorname{VAR}\left(S_{i}\right)$ and $\operatorname{CHAN}\left(\psi_{i}\right) \subseteq \operatorname{CHAN}\left(S_{i}\right)$.

We conclude this presentation of the proof system with the following three rules: 
CONSEQuence RULES

$$
\frac{\{S\}_{C} \psi_{1}, \psi_{1} \rightarrow \psi_{2}}{\{S\}_{C} \psi_{2}} \quad \frac{\{P\} \psi_{1}, \psi_{1} \rightarrow \psi_{2}}{\{P\} \psi_{2}}
$$

CONJUNCTION RULE

$$
\frac{\{S\}_{C} \psi_{1},\{S\}_{C} \psi_{2}}{\{S\}_{C} \psi_{1} \wedge \psi_{2}}
$$

\section{SOUNDNESS}

The soundness of the system is proved by induction on the length of the derivation. In fact, it suffices to show that the axioms are valid, and that the rules preserve validity, i.e., the validity of the conclusion of a rule follows from that of its premises. In this section we prove the soundness of the rule for sequential composition.

\section{LEMMA 5.1.:}

If $\vDash\left\{S_{1}\right\}_{C} \psi_{1}$ and $\vDash\left\{S_{2}\right\}_{C} \psi_{2}$ then $\vDash\left\{S_{1} ; S_{2}\right\}_{C} \psi_{1}\left[\psi_{2} / Y\right]$.

PROOF:

Take a $\gamma, \eta,<\tau, n>$ and $\theta \in \operatorname{CONT}\left(O(C)\left(S_{1} ; S_{2}\right),<\tau, n>\right)$. Furthermore, let $\theta=\llbracket \psi_{2} \rrbracket(\gamma)(\eta\{\theta / Y\})$. (Recall that for an arbitrary $\psi, \gamma, \eta:[\psi](\gamma)(\eta)=\{\langle\tau, n\rangle:\langle\tau, n\rangle, \gamma, \eta \vDash \psi\}$.) We have

$$
<\tau, n>, \gamma, \eta\{\theta / Y\} \vDash \psi_{1}\left[\psi_{2} / Y\right] \text { iff }<\tau, n>, \gamma, \eta\{\theta / Y\} \vDash \psi_{1}
$$

A proof of the above equivalence we omit, it is straightforward but tedious. As $\theta \in \operatorname{CONT}\left(O(C)\left(S_{1} ; S_{2}\right),<\tau, n>\right)$ there exist $n_{1}$ and $n_{2}$ such that

$$
\begin{aligned}
& n \leqslant n_{1} \leqslant \omega \text { and } \tau\left[n, n_{1}\right] \in O(C)\left(S_{1}\right), \\
& n_{1} \leqslant n_{2} \leqslant \omega \text { and } \theta=\left\{<\tau, n_{2}>\right\}, \text { and if } n_{1}<\omega \text { then } \tau\left[n_{1}, n_{2}\right] \in O(C)\left(S_{2}\right) .
\end{aligned}
$$

Let $\left.\theta^{\prime}=\left\{<\tau, n_{1}\right\rangle\right\}$. So we have $\theta^{\prime} \in \operatorname{CONT}\left(O(C)\left(S_{1}\right),<\tau, n>\right)$. From $\vDash\left\{S_{1}\right\}_{C} \psi_{1}$ we then infer $<\tau, n>, \gamma, \eta\left\{\theta^{\prime} / Y\right\} \vDash \psi_{1}$. Now suppose $n_{1}<\omega$ : From $\theta \in \operatorname{CONT}\left(O(C)\left(S_{2}\right),<\tau, n_{1}>\right)$ and $\vDash\left\{S_{2}\right\}_{C} \psi_{2}$ we infer $<\tau, n_{1}>, \gamma, \eta\{\theta / Y\} \vDash \psi_{2}$. So we have $\left.\theta^{\prime} \subseteq \llbracket \psi_{2}\right\rfloor(\gamma)\left(\eta\{\theta / Y\}\right.$ ) (if $n_{1}=\omega$ then $\theta^{\prime}=\varnothing$ ). From $<\tau, n>, \gamma, \eta\left\{\theta^{\prime} / Y\right\} \vDash \psi_{1}$ and the monotonicity of $\psi_{1}$ in $Y$ we conclude: $<\tau, n>, \gamma, \eta\{\theta / Y\} \vDash \psi_{1}$, or, equivalently, $\left\langle\tau, n>, \gamma, \eta \vDash \psi_{1}\left[\psi_{2} / Y\right]\right.$.

It is not difficult to see that for the rule for sequential composition to be sound it is necessary that $Y$ occurs positively in $\psi_{1}$. Consider the following: Let $S_{1}, S_{2} \equiv x:=x$ and $\psi_{1} \equiv \neg Y, \psi_{2} \equiv$ true, $C=(\{x\}, \varnothing)$. Then $\vDash\left\{S_{i}\right\}_{C} \psi_{i}$. Note that $\psi_{1}$ expresses that the execution of the statement $x:=x$ does not terminate immediately. But it is not the case that $\vDash\{x:=x ; x:=x\}_{C}$ false!

\section{COMPLETENESS}

In this section we prove that an arbitrary well-formed specification about a statement or a program is derivable using as additional axioms all the valid assertions (the underlying temporal theory). (Note that we assume the interpretation $I$ to be fixed.) We prove completeness by associating with each statement $S$ and context $C$ such that $C O N(S) \subseteq C$ an assertion $\psi$ such that $\psi$ defines $O(C)(S)$. First we will formalize this notion of an assertion defining the semantics of a statement.

DEFINITION 6.1:

Given the environments $\gamma$ and $\eta$ we define the extension of an assertion $\psi$, which we denote by $[\psi]_{\gamma, \eta}$, as follows:

$$
[\psi]_{\gamma, \eta}=\min \{(<\tau, n>, \theta): \theta \in \operatorname{CONT}(<\tau, n>) \wedge<\tau, n>, \gamma, \eta\{\theta / Y\} \vDash \psi\}
$$


where the operation min takes all the minimal elements with respect to the following ordering:

$$
(<\tau, n>, \theta) \leqslant\left(<\tau^{\prime}, m>, \theta^{\prime}\right) \text { iff } \tau=\tau^{\prime} \wedge n=m \wedge \theta \subseteq \theta^{\prime} .
$$

Note that $\theta \subseteq \theta^{\prime}$, for $\theta, \theta^{\prime} \in \operatorname{CONT}(<\tau, n>)$ iff $\theta=\theta^{\prime}$ or $\theta=\varnothing$.

We demand $\psi$ to be monotonic in $Y$, therefore we apply the operation $\min$ to the set of pairs $(<\tau, n\rangle, \theta)$, with $\theta \in \operatorname{CONT}(<\tau, n\rangle)$, such that $\psi$ holds in $\langle\tau, n\rangle$ when $Y$ is interpreted as $\theta$.

Definition 6.2:

Given the environments $\gamma$ and $\eta$ we define the set of sequences determined by an assertion $\psi$, which we denote by $O_{\gamma, \eta}(\psi)$, as follows:

$$
O_{\gamma, \eta}(\psi)=\left\{\tau[n, m]:(<\tau, n>, \theta) \in[\psi]_{\gamma, \eta}, \text { with } \theta=\{<\tau, m>\}\right\}
$$

Now $O_{\gamma, \eta}(\psi) \subseteq O(C)(S)$ for an arbitrary $\gamma, \eta$ formalizes the notion of $\psi$ defining the semantics of $S$ relative to the context $C$. We next prove that for an arbitrary statement $S$ and context $C$ (with $\operatorname{CON}(S) \subseteq C)$ there exists an assertion $\psi$ such that

$$
\vdash\{S\}_{C} \psi \text { and for every } \gamma, \eta \text { we have } O_{\gamma, \eta}(\psi) \subseteq O(C)(S) \text {. }
$$

Completeness then follows: Let $k\{S\}_{C} \phi,\{S\}_{C} \phi$ being a well-formed specification. Furthermore, let $\psi$ such that $\vdash\{S\}_{C} \psi$ and for every $\gamma, \eta$ we have $O_{\gamma, \eta}(\psi) \subseteq O(C)(S)$. As we have $\vDash\{S\}_{C} \phi$ by an application of the consequence rule it thus suffices to show that $k \psi \rightarrow \phi$. So let $\gamma, \eta,\langle\tau, n>, \theta \in \operatorname{CONT}(<\tau, n>) \quad$ such that $<\tau, n>, \gamma, \eta\{\theta / Y\} \vDash \psi$. First suppose that $(<\tau, n>, \theta) \in[\psi]_{\gamma, \eta}$, say $\theta=\{<\tau, m>\}$ : So we have $\tau[n, m] \in O_{\gamma, \eta}(\psi) \subseteq O(C)(S)$. By $\vDash\{S\}_{C} \phi$ we then infer that $<\tau, n>, \gamma, \eta\{\theta / Y\} \vDash \phi$. If on the other hand $\left(\langle\tau, n>, \theta) \notin[\psi]_{\gamma, \eta}\right.$ we have $(<\tau, n>, \varnothing) \in[\psi]_{\gamma, \eta}$. So we have $\tau[n, \omega] \in O_{\gamma, \eta}(\psi) \subseteq O(C)(S)$. Now by $k\{S\}_{C} \phi$ we infer that $\langle\tau, n>, \gamma, \eta\{\varnothing / Y\} \vDash \phi$. But $\phi$ is monotonic in $Y$ so we conclude $<\tau, n>, \gamma, \eta\{\theta / Y\} \vDash \phi$.

\section{LEMMA 6.1.:}

For an arbitrary statement $S$ and context $C$, with $\operatorname{CON}(S) \subseteq(C)$, there exists an assertion $\psi$ such that $\vdash\{S\}_{C} \psi,\{S\}_{C} \psi$ being a well-formed specification, and for every $\gamma, \eta$ we have $O_{\gamma, \eta}(\psi) \subseteq O(C)(S)$.

\section{PROOF:}

We show that for every statement $S$ and context $C$, with $\operatorname{CON}(S) \subseteq C$, there exists an assertion $\psi$ such that $\vdash\{S\}_{C} \psi$ and if $\langle\tau, n>, \gamma, \eta \vDash \psi(\gamma, \eta$ arbitrary) then for some $\theta=\{<\tau, m\rangle\} \in \operatorname{CONT}(<\tau, n\rangle)$, with $\theta \subseteq \eta(Y)$, we have $\tau[n, m] \in O(C)(S)$. This suffices: Let $(<\tau, n>, \theta) \in[\psi]_{\gamma, \eta}$, it then follows that for some $\theta^{\prime}=\left\{\langle\tau, m>\} \subseteq \theta\right.$ we have $\tau[n, m] \in O(C)(S)$. By $\vDash\{S\}_{C} \psi$ (which follows from $r\{S\}_{C} \psi$ and the soundness of the system) we have $<\tau, n>, \gamma, \eta\left\{\theta^{\prime} / Y\right\} \vDash \psi$. But $(<\tau, n>, \theta) \in[\psi]_{\gamma, \eta}$ so we conclude $\theta^{\prime}=\theta$.

The proof goes by induction on the complexity of $S$. We treat the cases of $S$ an atomic statement and of $S \equiv S_{1} ; S_{2}$.

$S$ atomic: We illustrate this case by a simple example. Let $S \equiv x:=y$ and $C=(\{x, y\}, \varnothing)$.

Now:

$$
r\{x:=y\}_{C} \psi_{1}
$$

by the invariance axiom, where $\psi_{1} \equiv L E T z_{1}=y I N z_{1}=y \quad U z_{1}=y \wedge Y N I$. Furthermore we have:

$$
r\{x:=y\}_{C} \psi_{2}
$$

by the assignment axiom, where $\psi_{2} \equiv L E T z_{2}=x I N z_{2}=x U x=y \wedge Y N I$. So we infer

$$
\vdash\{x:=y\}_{C} \psi_{1} \wedge \psi_{2}
$$

by the conjunction rule. Finally we conclude 


$$
\vdash\{x:=y\}_{C} \psi
$$

by the consequence rule, where $\psi \equiv L E T z_{1}, z_{2}=y, x I N z_{1}=y \wedge z_{2}=x \quad U z_{1}=y \wedge x=y \wedge Y N I$ (use the validity of the assertion $\square_{\neg} Y \vee(\neg Y U Y \wedge \square \neg Y)$, which expresses that the variable $Y$ denotes some unique continuation). Now it is straightforward to check that if $\langle\tau, n\rangle, \gamma, \eta \neq \psi$ then for some $\theta=\{<\tau, m>\} \subseteq \eta(Y)$ we have $\tau[n, m] \in O(C)(x:=y)$.

$S \equiv S_{1} ; S_{2}$ : The induction hypothesis gives us assertions $\psi_{1}$ and $\psi_{2}$ such that, among others, $\vdash\{S\}_{C} \psi_{1}$ and $r\left\{S_{2}\right\}_{C} \psi_{2}$. So by the rule for sequential composition we have $+\left\{S_{1} ; S_{2}\right\}_{C} \psi_{1}\left[\psi_{2} / Y\right]$. Let $\gamma, \eta,\langle\tau, n\rangle$ such that $\langle\tau, n\rangle, \gamma, \eta \vDash \psi_{1}\left[\psi_{2} / Y\right]$. We show that there exists a $n \leqslant m \leqslant \omega$ such that $\tau[n, m] \in O(C)(S)$ and $\{\langle\tau, m\rangle\} \subseteq \eta(Y)$ : Let $\Theta=\llbracket \psi_{2} \rrbracket(\gamma)(\eta)$. We have

$$
<\tau, n>, \gamma, \eta \vDash \psi_{1}\left[\psi_{2} / Y\right] \text { iff }<\tau, n>, \gamma, \eta\{\Theta / Y\} \vDash \psi_{1} \text {. }
$$

By the induction hypothesis we have for some $n \leqslant k \leqslant \omega$

$$
\{<\tau, k>\} \subseteq \Theta \text { and } \tau[n, k] \in O\left(S_{1}\right)(C) \text {. }
$$

If $k=\omega$ then $\tau[n, \omega] \in O(C)(S)$ and we are done $(\{<\tau, \omega\rangle\}=\varnothing)$. So suppose $k<\omega$ : By the induction hypothesis $\left(<\tau, k>, \gamma, \eta \vDash \psi_{2}\right)$ we have for some $k \leqslant m \leqslant \omega$ that

$$
\{<\tau, m>\} \subseteq \eta(Y) \text { and } \tau[k, m] \in O(C)\left(S_{2}\right) \text {. }
$$

By the definition of the semantics of statements it then follows that $\tau[n, m] \in O(C)\left(S_{1} ; S_{2}\right)$, which concludes the proof.

Now let $P \equiv S_{1}\|\cdots\| S_{n}$. We prove that an arbitrary valid specification of $P$ is derivable. It suffices to show that there exists an assertion $\psi$ such that for every $\gamma, \eta,\langle\tau, n\rangle$ if $\langle\tau, n\rangle, \gamma, \eta \neq \psi$ then $\tau[n, \omega] \in O(P)$ and $\vdash\{P\} \psi$. From this completeness easily follows: $\vDash\{P\} \phi$ implies $\vDash \psi \rightarrow \phi$ (note that as $Y$ does not occur in $\psi$ and $\phi$ we have $\vDash \psi \rightarrow \phi$ iff for every $\gamma, \eta,\langle\tau, n\rangle$ if $\langle\tau, n\rangle, \gamma, \eta \vDash \psi$ then $<\tau, n>, \gamma, \eta \vDash \phi)$. Now by the previous lemma there exist assertions $\psi_{i}$ such that for every $\gamma, \eta$ we have $\quad O_{\gamma, \eta}\left(\psi_{i}\right) \subseteq O\left(C_{i}\right)\left(S_{i} ;\right.$ halt $)$, with $C_{i}=\operatorname{CON}\left(S_{i}\right), \quad$ and $\quad f\left\{S_{i} ; \text { halt }\right\}_{C_{i}} \psi_{i}$. Let $<\tau, n>, \gamma, \eta \vDash \wedge_{i} \psi_{i}[$ false $/ Y]$, with $<\tau, n>, \gamma, \eta$ arbitrary. It then follows that $<\tau, n>, \gamma, \eta\{\varnothing / Y\} \vDash \psi_{i}, \quad$ or $\quad(<\tau, n>, \varnothing) \in\left[\psi_{i}\right]_{\gamma, \eta}$. As $O_{\gamma, \eta}\left(\psi_{i}\right) \subseteq O\left(C_{i}\right)\left(S_{i} ;\right.$ halt $)$ we have $\tau[n, \omega] \in O\left(C_{i}\right)\left(S_{i} ;\right.$ halt $)$, so we infer $\tau[n, \omega] \in O(P)$. Thus we can put $\psi \equiv \wedge_{i} \psi_{i}[$ false/Y].

\section{Nested Parallel.ism}

In this last section we show how to handle nested parallelism. One of the main problems in specifying nested parallelism, in a compositional manner, in temporal logic is how to describe the behaviour arising from executions of parallel statements in which one component terminates while some other components are still executing. (To define $S_{1} \| S_{2}$ in terms of $S_{i}$; halt, as we did in section 2, does not work because this generates only infinite computations so that we can not describe the semantics of statements like $\left(S_{1} \| S_{2}\right) ; S_{3}$.) To be able to describe such behaviour we extend our temporal language with quantification over propositional variables ([W]). Let $P R O P$ be a set of propositional variables with typical element $p$. States are extended with a component $P R O P \rightarrow B O O L$ which gives meaning to the propositional variables, $B O O L$ being the class of boolean values. The class of assertions is extended by the following rule.

$$
\psi::=\exists p \psi
$$

An assertion $\exists p \psi$ is interpreted as follows.

\section{DEFINITION 7.1.:}

We define: 
$<\tau, n>, \gamma, \eta \vDash \exists p \psi$ iff for some $\tau^{\prime}$ differing from $\tau$ only with respect to $p$ we have $\left\langle\tau^{\prime}, n\right\rangle, \gamma, \eta \vDash \psi$.

Here two sequences $\tau$ and $\tau^{\prime}$ are said to differ with respect to a propositional variable $p$ iff every two states $\tau(n)$ and $\tau^{\prime}(n)$ agree with respect to the sets VAR,CHAN and PROP\\{p\}. Next we extend our class of statements by the following rule:

$$
\begin{aligned}
& S::=S_{1} \| S_{2} \\
& \text { where } I N\left(S_{1}\right) \cap I N\left(S_{2}\right)=\operatorname{OUT}\left(S_{1}\right) \cap \operatorname{OUT}\left(S_{2}\right)=\operatorname{VAR}\left(S_{1}\right) \cap \operatorname{VAR}\left(S_{2}\right)=\varnothing
\end{aligned}
$$

$I N(S)(O U T(S))$ denotes the set of input (output) channels occurring in $S$. This restriction on channels generalizes the restriction as described in the first section. A program is now just a statement. To describe the semantics of statements of the form $S_{1} \| S_{2}$ we will use these propositional variables to signal the termination of the subprocesses executing the statements $S_{1}$ and $S_{2}$. We will associate with $S_{i}$ a variable $p_{i}$ such that $p_{i}$ is false during the execution of $S_{i}$ and becomes true when the execution of $S_{i}$ has terminated. To model this compositionally we extend our notion of a context. We define a context $C$ to be a triple $\left(V_{1}, V_{2}, V_{3}\right)$, where $V_{1} \subseteq V A R, V_{2} \subseteq C H A N$, and $V_{3} \subseteq P R O P$. When we associate a context $C=\left(V_{1}, V_{2}, V_{3}\right)$ with a statement $S$, as will be the case in a specification $\{S\}_{C} \psi$, then the variables of $V_{3}$ are supposed to signal the termination of those processes executing a statement of which $S$ is a substatement. This implies that during the execution of $S$ all the variables of $V_{3}$ must be false. This is formalized by the following axiom:

- $\{S\}_{C} \rightarrow p U Y$

where, assuming $C=\left(V_{1}, V_{2}, V_{3}\right), p \in V_{3}$. This axiom states that while $S$ has not terminated the process, the termination of which is signaled by $p$, has not terminated either. Note that $Y$ marks the termination of $S$.

To describe the termination protocol we introduce the auxiliary statements $p ?$ and $p:=$ ? the semantics of which is given by the following specifications:

- $\{p ?\}_{C} p \wedge Y$.

So $p$ ? tests if $p$ holds and terminates, otherwise it aborts.

- $\{p:=?\}_{C} \neg p U Y$.

This specification describes the effect of assigning a arbitrary value to $p$, assuming $p$ to be true initially. Let $p_{1}$ signal the termination of $S_{1}$ and $p_{2}$ that of $S_{2}$, the termination protocol then is described by the parallel execution of the statements $p_{i} ? ; W H I L E \rightarrow p_{j} D O p_{j}:=? O D, i \not g j$.

Now we can formulate the following proof rule for nested parallelism:

$$
\text { - } \frac{\left\{S_{i}\right\}_{C_{i}} \psi_{i},\left\{p_{i} ? ; \text { WHILE } \neg p_{j} D O p_{j}:=? O D\right\}_{C_{i}} \phi_{i}}{\left\{S_{1} \| S_{2}\right\}_{C} \exists p_{1}, p_{2}\left(\psi_{1}\left[\phi_{1} / Y\right] \wedge \psi_{2}\left[\phi_{2} / Y\right]\right)}
$$

where, assuming $C=\left(V_{1}, V_{2}, V_{3}\right)$, we have $C_{i}=\left(V_{1} \backslash \operatorname{VAR}\left(S_{j}\right), V_{2} \cap C H A N\left(S_{i}\right), V_{3} \cup\left\{p_{i}\right\}\right)$, with $p_{1}, p_{2}$ fresh propositional variables (neither occurring in $\psi_{i}$, free or bound, nor in $V_{3}$ ) and $i \neq j \in\{1,2\}$. Furthermore, $C_{i}^{\prime}=\left(V_{1} \backslash \operatorname{VAR}\left(S_{j}\right), V_{2} \cap \operatorname{CHAN}\left(S_{i}\right), V_{3}\right)$.

First note that the first two components of $C_{i}$ quarantee freedom from interference: $\psi_{i}$ will neither be allowed to mention variables of $S_{j}$ nor those channels of $S_{j}$ which do not connect $S_{j}$ with $S_{i}$. Furthermore by adding the variable $p_{i}$ to $V_{3}$ we know by the above axiom that during the execution of $S_{i}$ $p_{i}$ will be false. Note that as $p_{j}$ is not a member of $V_{3}$ nothing can be said about this variable by $\psi_{i}$. 
We abstract from the control information encoded by the variables $p_{1}$ and $p_{2}$ by existantially quantifying them in the conclusion of the rule.

A proof of the soundness and completeness of this proof system for nested parallelism goes along similar lines as the one given. In these proofs we use a slightly different notion of a continuation, that is, we had to modify the interpretation of the variable $Y$ to make it consistent with the quantification over propositional variables. This different interpretation of the notion of a continuation can be axiomatized in a similar way as the old one.

\section{Conclusion}

We have developed a new method for specifying in a compositional manner, in a temporal logic with as sole temporal operator the until, without making use of location predicates, sequential composition, its iterated version, and its interaction with (nested) parallelism, in the context of a CSP-like language. We did so by specifying statements with respect to some possible continuation, denoted by some predicate variable. The reasoning process about these continuations can be easily formalized in the logic. We proved this method to be sound and complete with respect to a denotational semantics which associates with each program a set of sequences of states. The rules and axioms of the proof system closely mirror the semantical operations describing the different ways programs can be constructed.

\section{ACKNOWLEDGEMENT}

Discussions with Pierre America, Jaco de Bakker, Joost Kok, John-Jules Meyer, Jan Rutten and Erik de Vink contributed to the formulation of the ideas presented in this paper, the emergence of which was stimulated by discussions with Ruurd Kuiper. The idea of the LET . construct is due to Pierre America.

\section{REFERENCES}

[BKP1] H. Barringer, R. Kuiper, A. Pnueli (1984). Now you may compose temporal logic specifications, Proc. 16th ACM Symp. on Theory of Computing, pp. 51-63.

[BKP2] H. Barringer, R. Kuiper, A. Pnueli (1986) A really abstract concurrent model and its temporal logic, Proc. 13th ACM Symp. on Principles of Programming Languages, pp.173-183.

[D] E.W. Dijkstra (1976). A discipline of programming, Prentice Hall.

[H] C.A.R. Hoare (1981). Communicating sequential processes, CACM, Vol. 21, No. 8, pp. 666-677.

[HKP] D. Harel, D. Kozen, R. Parikh (1980). Process logic: expressiveness, decidability, completeness, 21 th Symp. on Foundations of Comp. Sc., Syracus, New York, pp. 129-142.

[HMM] J. Halpern, Z. Manna, B. Moszkowski (1983). A hardware semantics based on temporal intervals, Automata Languages and Programming, 10th Colloquium, No. 154, pp. 278-291.

[L1] L. Lamport (1985). An axiomatic semantics of concurrent programming languages, Logics and Models of Concurrent Systems, edited by K.R. Apt, Springer-Verlag Berlin Heidelberg.

[L2] L. Lamport (1980). The Hoare logic of concurrent programs, Acta Informatica, Vol. 14, pp 21-37.

[MP] Z. Manna, A. Pnueli (1983). How to cook a temporal proof system for your pet language, Proc. 


\section{0th ACM POPL.}

[NGO] V. Nguyen, D. Gries, S. Owicki (1985), A model and temporal proof system for network of processes, 12th ACM Symp. on Principles of Programming, pp. 121-131.

[OG] S. Owicki, D. Gries (1976), An axiomatic proof technique for concurrent parallel programs, Acta Informatica, No. 6, pp. 319-340.

[P] A. Prior (1967). Past, present and future, Oxford University Press, London.

[Pn] A. Pnueli (1977). The temporal logic of programs, 18th IEEE Symp. Foundations of Computer Science, pp.46-57.

[T] A. Tarski (1955), A lattice-theoretical fixpoint theorem and its applications, Pacific J. Math., No. 5, pp. 285-309.

[W] P. Wolper (1981), Temporal logic can be more expressive, 22th Annual Symp. on Foundations of Computer Science, pp. 340-347.

[ZRE] J. Zwiers, W.P. de Roever, P. van Emde Boas (1985). Compositionality and concurrent networks: soundness and completeness of a proof system, Automata, Languages and Programming (ICALP), 12th Colloquium, Nafplion, Greece, Vol. 194, pp. 509-519. 
In this series appeared :

\begin{tabular}{|c|c|c|}
\hline No. & Author(s) & Title \\
\hline $85 / 01$ & R.H. Mak & The formal specification and derivation of CMOS-circuits. \\
\hline $85 / 02$ & W.M.C.J. van Overveld & On arithmetic operations with $\mathrm{M}$-out-of- $\mathrm{N}$-codes. \\
\hline $85 / 03$ & W.J.M. Lemmens & Use of a computer for evaluation of flow films. \\
\hline $85 / 04$ & $\begin{array}{l}\text { T. Verhoeff } \\
\text { H.M.L.J.Schols }\end{array}$ & $\begin{array}{l}\text { Delay insensitive directed trace structures satisfy the foam } \\
\text { the foam rubber wrapper postulate. }\end{array}$ \\
\hline $86 / 01$ & R. Koymans & Specifying message passing and real-time systems. \\
\hline $86 / 02$ & $\begin{array}{l}\text { G.A. Bussing } \\
\text { K.M. van Hee } \\
\text { M. Voorhoeve }\end{array}$ & $\begin{array}{l}\text { ELISA, A language for formal specification of } \\
\text { information systems. }\end{array}$ \\
\hline $86 / 03$ & Rob Hoogerwoord & Some reflections on the implementation of trace structures. \\
\hline $86 / 04$ & $\begin{array}{l}\text { G.J. Houben } \\
\text { J. Paredaens }\end{array}$ & $\begin{array}{l}\text { The partition of an information system in several } \\
\text { systems. }\end{array}$ \\
\hline
\end{tabular}

K.M. van Hee

$\begin{array}{ll}86 / 05 & \begin{array}{l}\text { J.L.G. Dietz } \\ \text { K.M. van Hee }\end{array} \\ 86 / 06 & \text { Tom Verhoeff } \\ 86 / 07 & \begin{array}{l}\text { R. Gerth } \\ \text { L. Shira }\end{array} \\ 86 / 08 & \begin{array}{l}\text { R. Koymans } \\ \text { R.K. Shyamasundar } \\ \text { W.P. de Roever } \\ \text { R. Gerth } \\ \text { S. Arun Kumar }\end{array} \\ 86 / 09 & \begin{array}{l}\text { C. Huizing } \\ \text { R. Gerth } \\ \text { W.P. de Roever }\end{array} \\ \end{array}$

$86 / 10$ J. Hooman

86/11 W.P. de Roever

$86 / 12$ A. Boucher

R. Gerth

86/13 R. Gerth W.P. de Roever
A framework for the conceptual modeling of discrete dynamic systems.

Nondeterminism and divergence created by concealment in CSP.

On proving communication closedness of distributed layers.

Compositional semantics for real-time distributed computing (Inf.\&Control 1987).

Full abstraction of a real-time denotational semantics for an OCCAM-like language.

A compositional proof theory for real-time distributed message passing.

Questions to Robin Milner - A responder's commentary (IFIP86).

A timed failures model for extended communicating processes.

Proving monitors revisited: a first step towards WXrifying object oriented systems (Fund. Informatica 

$86 / 14$
R. Koymans
87/01 R. Gerth

87/02 Simon J. Klaver Chris F.M. Verberne

87/03 G.J. Houben J.Paredaens

87/04 T.Verhoeff

87/05 R.Kuiper

87/06 R.Koymans

87/07 R.Koymans

87/08 H.M.J.L. Schols

87/09 J. Kalisvaart

L.R.A. Kessener

W.J.M. Lemmens

M.L.P. van Lierop

F.J. Peters

H.M.M. van de Wetering

$87 / 10 \quad$ T.Verhoeff

87/11 P.Lemmens

87/12 K.M. van Hee and A.Lapinski

87/13 J.C.S.P. van der Woude

87/14 J. Hooman

$87 / 15$ C. Huizing

R. Gerth

W.P. de Roever

87/16 H.M.M. ten Eikelder J.C.F. Wilmont

87/17 K.M. van Hee G.-J.Houben J.L.G. Dietz
Specifying passing systems requires extending temporal logic.

On the existence of sound and complete axiomati zations of the monitor concept.

Federatieve Databases.

A formal approach to distributed information systems.

Delay-insensitive codes - An overview.

Enforcing non-determinism via linear time temporal logic specification.

Temporele logica specificatie van message passing en real-time systemen (in Dutch).

Specifying message passing and real-time systems with real-time temporal logic.

The maximum number of states after projection.

Language extensions to study structures for raster graphics.

Three families of maximally nondeterministic automata.

Eldorado ins and outs. Specifications of a data base management toolkit according to the functional model.

OR and AI approaches to decision support systems.

Playing with pattems - searching for strings.

A compositional proof system for an occam-like real-time language.

A compositional semantics for statecharts.

Normal forms for a class of formulas.

Modelling of discrete dynamic systems framework and examples. 
87/18 C.W.A.M. van Overveld

87/19 A.J.Seebregts

87/20 G.J. Houben

J. Paredaens

$87 / 21$ R. Gerth

M. Codish

Y. Lichtenstein

E. Shapiro

$88 / 01$ T. Verhoeff

88/02 K.M. van Hee

G.J. Houben

L.J. Somers

M. Voorhoeve

$88 / 03$ T. Verhoeff

88/04 G.J. Houben

J.Paredaens

D.Tahon

88/05 K.M. van Hee

G.J. Houben

L.J. Somers

M. Voorhoeve

88/06 H.M.J.L. Schols

$88 / 07$ C. Huizing

R. Gerth

W.P. de Roever

88/08 K.M. van Hee

G.J. Houben

L.J. Somers

M. Voorhoeve

88/09 A.T.M. Aerts

K.M. van Hee

88/10 J.C. Ebergen

88/11 G.J. Houben

J.Paredaens

88/12 A.E. Eiben

$88 / 13$ A. Bijlsma

88/14 H.M.M. ten Eikelder

R.H. Mak
An integer algorithm for rendering curved surfaces.

Optimalisering van file allocatie in gedistribueerde database systemen.

The $\mathrm{R}^{2}$-Algebra: An extension of an algebra for nested relations.

Fully abstract denotational semantics for concurrent PROLOG.

A Parallel Program That Generates the Möbius Sequence.

Executable Specification for Information Systems.

Settling a Question about Pythagorean Triples.

The Nested Relational Algebra: A Tool to Handle Structured Information.

Executable Specifications for Information Systems.

Notes on Delay-Insensitive Communication.

Modelling Statecharts behaviour in a fully abstract way.

A Formal model for System Specification.

A Tutorial for Data Modelling.

A Formal Approach to Designing Delay Insensitive Circuits.

A graphical interface formalism: specifying nested relational databases.

Abstract theory of planning.

A unified approach to sequences, bags, and trees.

Language theory of a lambda-calculus with recursive types. 


\begin{tabular}{|c|c|}
\hline $88 / 15$ & $\begin{array}{l}\text { R. Bos } \\
\text { C. Hemerik }\end{array}$ \\
\hline $88 / 16$ & $\begin{array}{l}\text { C.Hemerik } \\
\text { J.P.Katoen }\end{array}$ \\
\hline $88 / 17$ & $\begin{array}{l}\text { K.M. van Hee } \\
\text { G.J. Houben } \\
\text { L.J. Somers } \\
\text { M. Voorhoeve }\end{array}$ \\
\hline $88 / 18$ & $\begin{array}{l}\text { K.M. van Hee } \\
\text { P.M.P. Rambags }\end{array}$ \\
\hline $88 / 19$ & $\begin{array}{l}\text { D.K. Hammer } \\
\text { K.M. van Hee }\end{array}$ \\
\hline $88 / 20$ & $\begin{array}{l}\text { K.M. van Hee } \\
\text { L. Somers } \\
\text { M.Voorhoeve }\end{array}$ \\
\hline $89 / 1$ & E.Zs.Lepoeter-Molnar \\
\hline $89 / 2$ & $\begin{array}{l}\text { R.H. Mak } \\
\text { P.Struik }\end{array}$ \\
\hline $89 / 3$ & $\begin{array}{l}\text { H.M.M. Ten Eikelder } \\
\text { C. Hemerik }\end{array}$ \\
\hline $89 / 4$ & $\begin{array}{l}\text { J.Zwiers } \\
\text { W.P. de Roever }\end{array}$ \\
\hline $89 / 5$ & $\begin{array}{l}\text { Wei Chen } \\
\text { T.Verhoeff } \\
\text { J.T.Udding }\end{array}$ \\
\hline $89 / 6$ & T.Verhoeff \\
\hline $89 / 7$ & P.Struik \\
\hline $89 / 8$ & $\begin{array}{l}\text { E.H.L.Aarts } \\
\text { A.E.Eiben } \\
\text { K.M. van Hee }\end{array}$ \\
\hline $89 / 9$ & $\begin{array}{l}\text { K.M. van Hee } \\
\text { P.M.P. Rambags }\end{array}$ \\
\hline $89 / 10$ & S.Ramesh \\
\hline $89 / 11$ & S.Ramesh \\
\hline $89 / 12$ & $\begin{array}{l}\text { A.T.M.Aerts } \\
\text { K.M. van Hee }\end{array}$ \\
\hline
\end{tabular}

An introduction to the category theoretic solution of recursive domain equations.

Bottom-up tree acceptors.

Executable specifications for discrete event systems.

Discrete event systems: concepts and basic results.

Fasering en documentatie in software engineering.

EXSPECT, the functional part.

Reconstruction of a 3-D surface from its normal vectors.

A systolic design for dynamic programming.

Some category theoretical properties related to a model for a polymorphic lambda-calculus.

Compositionality and modularity in process specification and design: A trace-state based approach.

Networks of Communicating Processes and their (De-)Composition.

Characterizations of Delay-Insensitive Communication Protocols.

A systematic design of a paralell program for Dirichlet convolution.

A general theory of genetic algorithms.

Discrete event systems: Dynamic versus static topology.

A new efficient implementation of CSP with output guards.

Algebraic specification and implementation of infinite processes.

A concise formal framework for data modeling. 
89/13 A.T.M.Aerts

K.M. van Hee

M.W.H. Hesen

89/14 H.C.Haesen

89/15 J.S.C.P. van der Woude

89/16 A.T.M.Aerts

K.M. van Hee

89/17 M.J. van Diepen

K.M. van Hee

90/1 W.P.de Roever-H.Barringer C.Courcoubetis-D.Gabbay R.Gerth-B.Jonsson-A.Pnueli M.Reed-J.Sifakis-J.Vytopil P.Wolper

90/2 K.M. van Hee P.M.P. Rambags

90/3 R. Gerth

90/4 A. Peeters

90/5 J.A. Brzozowski

J.C. Ebergen

90/6 A.J.J.M. Marcelis

90/7 A.J.J.M. Marcelis

90/8 M.B. Josephs

90/9 A.T.M. Aerts

P.M.E. De Bra

K.M. van Hee

90/10 M.J. van Diepen

K.M. van Hee

90/11 P. America

F.S. de Boer

90/12 P.America

F.S. de Boer

90/13 K.R. Apt

F.S. de Boer

E.R. Olderog

90/14 F.S. de Boer

90/15 F.S. de Boer
A program generator for simulated annealing problems.

ELDA, data manipulatie taal.

Optimal segmentations.

Towards a framework for comparing data models.

A formal semantics for $Z$ and the link between $\mathrm{Z}$ and the relational algebra.

Formal methods and tools for the development of distributed and real time systems, pp. 17.

Dynamic process creation in high-level Petri nets, pp. 19.

Foundations of Compositional Program Refinement - safety properties - , p. 38.

Decomposition of delay-insensitive circuits, p. 25 .

On the delay-sensitivity of gate networks, p. 23.

Typed inference systems : a reference document, p. 17.

A logic for one-pass, one-attributed grammars, p. 14.

Receptive Process Theory, p. 16.

Combining the functional and the relational model, p. 15.

A formal semantics for $Z$ and the link between $Z$ and the relational algebra, p. 30 . (Revised version of CSNotes 89/17).

A proof system for process creation, p. 84 .

A proof theory for a sequential version of POOL, p. 110.

Proving termination of Parallel Programs, p. 7.

A proof system for the language POOL, p. 70.

Compositionality in the temporal logic of concurrent systems, p. 17. 
90/16 F.S. de Boer

C. Palamidessi

90/17 F.S. de Boer

C. Palamidessi
A fully abstract model for concurrent logic languages, p. 23.

On the asynchronous nature of communication in concurrent logic languages: a fully abstract model based on sequences, p. 29. 